\title{
Use of sildenafil or other phosphodiesterase inhibitors and risk of melanoma
}

\author{
Anton Pottegård ${ }^{*}, 1$ Sigrún Alba Johannesdottir Schmidt ${ }^{2}$, Anne Braae Olesen ${ }^{3}$, Ninah Achacoso ${ }^{4}$, \\ Stephen K Van Den Eeden ${ }^{4,5}$, Jesper Hallas ${ }^{1}$, Henrik Toft Sørensen ${ }^{2}$, Søren Friis ${ }^{6}$ and Laurel A Habel ${ }^{4}$ \\ ${ }^{1}$ Clinical Pharmacology and Pharmacy, Department of Public Health, University of Southern Denmark, Odense, Denmark; \\ ${ }^{2}$ Department of Clinical Epidemiology, Aarhus University Hospital, Aarhus, Denmark; ${ }^{3}$ Department of Dermato-Venerology, \\ Aarhus University Hospital, Aarhus, Denmark; ${ }^{4}$ Division of Research, Kaiser Permanente Northern California, Oakland, CA, USA; \\ ${ }^{5}$ Department of Urology, UCSF, San Francisco, CA, USA and ${ }^{6}$ Danish Cancer Society Research Center, Danish Cancer Society, \\ Copenhagen, Denmark
}

Background: Phosphodiesterase 5A inhibitors (PDEls), a common treatment for erectile dysfunction, were recently linked to an increased risk of melanoma.

Methods: We conducted two parallel case-control studies, using the Danish Nationwide Health Registries (DNHR) and the Kaiser Permanente Northern California (KPNC) electronic health records. Identifying men with histologically verified melanoma (cases) matched on birth year to 10 cancer-free controls, we estimated odds ratios (OR) for melanoma associated with high use of PDEls $(\geqslant 100$ tablets filled), adjusting for available confounders.

Results: We identified 7045 DNHR and 2972 KPNC cases with invasive melanoma. The adjusted OR for invasive melanoma associated with high PDEl use was 1.22 (95\% confidence interval $(\mathrm{Cl}), 0.99-1.49)$ in DNHR and 0.95 (95\% Cl, 0.78-1.14) in KPNC. Odds ratios were highest for localised invasive melanoma in DNHR (OR, 1.21) and melanoma in situ in KPNC (OR, 1.15), and lowest for non-localised disease in both populations (ORs 0.75 and 0.61 , respectively). The increased ORs were slightly attenuated upon adjustment for markers of health-care utilisation.

Conclusions: We found little evidence for a causal association between PDEl use and risk of melanoma. The marginally increased risk of early stage disease likely resulted from more frequent health-care contacts among PDEI users.

Phosphodiesterase 5A inhibitors (PDEIs) are first-line treatment for erectile dysfunction (Ghofrani et al, 2006), a common condition, especially among elderly men (Shamloul and Ghanem, 2013). As population longevity increases, the overall prevalence of erectile dysfunction is expected to rise markedly, affecting more than 300 million men worldwide by 2025 (Shamloul and Ghanem, 2013). Any serious adverse effects associated with PDEI use are therefore of major public health concern.

In 2014, a cohort study conducted by Li et al (2014) among US male health professionals found that self-reported use of the PDEI sildenafil was associated with an increased risk of melanoma (hazard ratio, 1.92; 95\% confidence interval (CI), 1.14-3.22). However, this study lacked important data on timing, duration and dosing of PDEIs, tumour stage, and use of PDEIs other than sildenafil (Li et al, 2014).

An increased melanoma risk associated with PDEI use is biologically plausible. During the recent decade, knowledge of melanoma pathogenesis has improved, revealing that over $50 \%$ of melanomas contain activating mutations in BRAF (OMIM ${ }^{*}$ 164757) (Gray-Schopfer et al, 2007; Bollag et al, 2012; Hauschild et al, 2012). The downstream effect of BRAF activation is suppression of PDE5A, and the suppression of PDE5A stimulates melanoma cell invasion and metastasis (Arozarena et al, 2011). It is thus plausible that direct pharmacological inhibition of PDE5A may increase the risk of developing melanoma (Arozarena et al, 2011).

*Correspondence: A Pottegård; E-mail: apottegaard@health.sdu.dk

Received 26 April 2016; revised 20 July 2016; accepted 21 July 2016; published online 16 August 2016

(c) 2016 Cancer Research UK. All rights reserved 0007-0920/16

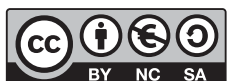

B) $C \cap$ 
Three additional studies of PDEIs and melanoma risk have subsequently been published. The first, conducted in Sweden (Loeb et al, 2015), found an increased risk for early stage disease (in situ and stage I), but a decreased risk for late-stage disease. In addition, there was no evidence of a dose response, although the highest category of exposure was modest. The next two studies were conducted in the UK CPRD (Lian et al, 2016; Matthews et al, 2016), and like the study by Li et al (2014), did not report findings by tumour stage. The one study reporting higher categories of exposure did not observe a dose response (Matthews et al, 2016). The findings of each these studies, although not consistently reproduced, led authors to question the causality of observed associations.

Additional data are clearly needed on higher levels of PDEI use, given these higher exposures are more likely to be aetiologically relevant for cancer risk. It also is important to further examine potential differences by stage, as well as examine associations in populations with different patterns of PDEI use and different prevalences of major risk factors such as sun exposure. To address these needs, we performed two parallel case-control studies employing nationwide data from the Danish Nationwide Health Registries (DNHR) and from electronic health records at Kaiser Permanente Northern California (KPNC).

\section{MATERIALS AND METHODS}

We conducted two independent case-control control studies using DNHR and KPNC data. In our primary analyses, we compared use of PDEIs among men diagnosed with invasive melanoma (cases) to that of cancer-free men (controls) to estimate odds ratios (ORs) for melanoma associated with PDEI use.

Data sources. As medical care in Denmark is provided by the National Health Authorities, the DNHR allow population-based registry linkage studies covering all Danish inhabitants $(\approx 5.6$ million). We obtained data from five Danish Nationwide Registries: the Danish Cancer Registry (Gjerstorff, 2011), the National Prescription Registry (Kildemoes et al, 2011), the National Registry of Patients (Schmidt et al, 2015), the Danish Education Registries (Jensen and Rasmussen, 2011), and the Danish Civil Registration System (Pedersen, 2011; Schmidt et al, 2014). Further information on these data sources is provided in Supplementary Appendix A. We linked data using the unique personal identification number, assigned to all Danish residents since 1968 (Pedersen, 2011).

Kaiser Permanente Northern California is an integrated healthcare delivery system providing comprehensive inpatient and outpatient care, including pharmacy services, to over 3.6 million current members, comprising about $30 \%$ of residents of areas served around San Francisco Bay and the Central Valley of California. We used the KPNC Cancer Registry, the KPNC pharmacy database, and other electronic clinical databases at KPNC providing data on hospitalisations and outpatient encounters (Selby et al, 2005). Additional information on the KPNC data sources is provided in Supplementary Appendix B. We linked data using a unique medical record number assigned to all health plan members.

Melanoma cases and population controls. We identified all men with a first diagnosis of primary invasive melanoma between 1 January 2000 and 31 December 2012 in the DNHR and between 1 January 2000 and 30 June 2014 in the KPNC database. At KPNC, we also identified men diagnosed with melanoma in situ during the same period. We used the date of diagnosis as the index date. To ensure the validity of our case material, we restricted the case populations to men with histologically confirmed tumours. Exclusion criteria was (i) age $<18$ years or age $>84$ years at diagnosis, (ii) $<5$ years of continuous follow up prior to the index date, (iii) previous cancer diagnosis (except non-melanoma skin cancer), (iv) dermatological conditions predisposing to melanoma (xeroderma pigmentosum, congenital non-neoplastic nevi, and, in DNHR, additionally dysplastic nevus syndrome and benign melanocytic nevi), and (v) conditions with acquired immunosuppression (human immunodeficiency virus infection and organ transplantation). As $<5 \%$ of melanomas are diagnosed in nonwhites, and membership of KPNC is racially/ethnically diverse, we also excluded (vi) non-white men within the KPNC. For additional details on exclusion criteria, see Supplementary Appendix C.

Within each study population, we used risk-set sampling to match 10 male population controls to each case by birth year, applying the same exclusion criteria as for cases. Further, to ensure similar availability of medication use and other health data for cases and controls at KPNC, we matched on a length of continuous health plan membership (in exact days). Controls were assigned an index date identical to that of the corresponding case. Cases were eligible for sampling as controls before their melanoma diagnosis. Thus, the calculated ORs provide unbiased estimates of the corresponding incidence rate ratios that would have emerged in cohort studies conducted in the underlying source populations (Rothman et al, 2008).

Exposure definition. Our primary exposure was use of PDEIs. Ever use of PDEIs was defined as two or more filled prescriptions for any PDEI prior to the index date, while non-use was defined as none or one filled prescription. High use of PDEIs was defined as having filled prescriptions equivalent to $\geqslant 100$ tablets prior to the index date. We also constructed categories of cumulative use based on number of tablets $(<20,20-49,50-99,100-199,200-499$, and $500+)$. In all definitions, we disregarded prescriptions filled within the year before the index date, as recent exposure is unlikely to be causally associated with cancer development (Burstein and Schwartz, 2008). All measures of exposure, including cut-offs, were selected prior to the conduct of the study. Marketing dates for single PDEIs in Denmark and the USA are provided in Supplementary Appendix D.

Statistical analysis. We used conditional logistic regression to compute ORs for melanoma associated with ever or high use of PDEI, and with categories of number of tablets (as above). In all analyses, non-use of PDEIs constituted the reference group.

We adjusted for several covariates known or suspected to be associated with either erectile dysfunction or melanoma risk. A full account of this adjustment is provided in Supplementary Appendix E.

We further conducted a number of pre-planned subgroup and sensitivity analyses. These analyses are detailed in Supplementary Appendix F.

Other. Analyses were performed using Stata Release 14.1 (StataCorp, College Station, TX, USA) for DNHR data and SAS version 9.3 (SAS Institute Inc., SAS Campus Drive, NC, USA) for KPNC data. The study was approved by the Danish Data Protection Agency. According to Danish law, studies based solely on registry data do not require approval from an Ethics Review Board (Thygesen et al, 2011). The KPNC Institutional Review Board approved the study.

\section{RESULTS}

We identified 8871 and 6210 cases with invasive melanoma in the DNHR and the KPNC database, respectively. The exclusions left 7045 and 2972 cases in the two study populations (flowcharts presented in Supplementary Results I). Baseline characteristics for cases and matched controls in each study population are shown in Table 1. 
In the DNHR, $6.4 \%(n=448)$ of cases were ever users of PDEIs, whereas $1.6 \%(n=113)$ were high users $(\geqslant 100$ tablets $)$. The corresponding prevalences for the KPNC population were 19.1 $(n=568)$ and $4.5 \%(n=133)$. In the DNHR, sildenafil was the most commonly used PDEI (comprising 70\% of all prescriptions among controls) followed by tadalafil (27\%), whereas sildenafil $(63 \%)$ and vardenafil $(35 \%)$ were the most common PDEIs in the KPNC population.

In the DNHR, the adjusted OR for melanoma was 1.06 (95\% CI, 0.96-1.18) for ever use and 1.22 (95\% CI, 0.99-1.49) for high use of PDEIs (Table 2). The corresponding ORs in the KPNC database were 1.01 (95\% CI, 0.91-1.12) and 0.95 (95\% CI, 0.78-1.14) (Table 2). Dose-response analyses by cumulative number of PDEI tablets yielded estimates close to unity in both study populations, except for ORs of 1.44 (95\% CI, 1.04-1.98) and 1.47 (95\% CI, 0.75-2.89) for use of 200-499 and 500 + tablets, respectively, in the DNHR (Table 2). Test for trend did not reach statistical significance in either database.

Results for the association between high use of PDEIs and melanoma according to clinical stage at diagnosis are shown in Table 3. Within the DNHR, the OR was 1.21 (95\% CI, 0.95-1.54) for localised melanoma and 0.75 (95\% CI, 0.32-1.75) for non-localised melanoma. The corresponding ORs for the KPNC population were 0.99 (95\% CI, 0.81-1.21) and 0.61 (95\% CI, 0.30-1.23).

Analyses of individual types of PDEIs within the DNHR yielded ORs for melanoma of 2.05 (95\% CI, 1.10-3.84) for use of 200-499 tablets of tadalafil and of 1.44 (95\% CI, 0.99-2.11) and 1.39 $(0.58-3.32)$ for $200-499$ and $500+$ tablets of sildenafil, respectively (Supplementary Results II). Within the KPNC population, use of $500+$ tablets of sildenafil was associated with an OR of 2.50 (95\% CI, 0.91-6.88), whereas use of 200-499 tablets of vardenafil revealed an OR of 1.38 (95\% CI, 0.88-2.16) (Supplementary Results II). Neither of these results reached statistical significance in tests for trend.

Defining exposure by doses of PDEI instead of number of tablets did not influence the overall associations (Supplementary Results III). However, within the DNHR, use of more than 500 doses of PDEIs returned an OR of 1.85 (95\% CI, 1.18-2.90). The corresponding OR was 1.22 (95\% CI 0.89-1.67) for KPNC and tests for trend did not reach statistical significance in either setting.

Among 2184 cases with melanoma in situ and 21582 controls in the KPNC database (for flowchart, see Supplementary Results IV), we observed an adjusted OR of 1.15 (95\% CI, 0.95-1.41), with no apparent dose-response pattern (complete results are presented in Supplementary Results V).

In the KPNC population, adjustment for number of ambulatory visits attenuated slightly the ORs for both invasive and in situ melanoma with high use of PDEI (invasive melanoma: from 0.95 to 0.90 ; in situ melanoma: from 1.15 to 1.09) (Supplementary Results V). Similarly, supplementary analyses in DNHR showed that educational level, a determinant of health-care utilisation, constituted the most influential covariate in the adjusted analyses reducing the overall OR from 1.28 to 1.24 , whereas adjustment for the remaining confounders only reduced the OR from 1.28 to 1.26 (data not shown). The generally marginal confounding was in accordance with the high degree of similarity in characteristics of cases and controls (Table 1).

Analyses according to subgroups defined by age, comorbidities, and concomitant medication had only limited influence on the OR estimates (Supplementary Results VI).

Finally, we observed a slightly stronger association for high use of PDEIs when using a 2-year lag time in the DNHR (OR, 1.28)

Table 1. Characteristics of invasive melanoma cases and their matched controls in the DNHR and the KPNC database

\begin{tabular}{|c|c|c|c|c|}
\hline & \multicolumn{2}{|c|}{ DNHR } & \multicolumn{2}{|c|}{ KPNC } \\
\hline & $\begin{array}{c}\text { Cases } \\
(n=7045)\end{array}$ & $\begin{array}{c}\text { Controls } \\
(n=70450)\end{array}$ & $\begin{array}{c}\text { Cases } \\
(n=2972)\end{array}$ & $\begin{array}{c}\text { Controls } \\
(n=29307)\end{array}$ \\
\hline Age, median (IQR, years) & $61(49-70)$ & $61(49-70)$ & $64(55-73)$ & $64(55-73)$ \\
\hline $\begin{array}{l}\text { Use of PDEls } \\
\text { Non-use } \\
\text { Ever use } \\
\text { High use ( } \geqslant 100 \text { tablets) }\end{array}$ & $\begin{array}{c}6597(93.6 \%) \\
448(6.4 \%) \\
113(1.6 \%)\end{array}$ & $\begin{array}{c}66295(94.1 \%) \\
4155(5.9 \%) \\
879(1.2 \%)\end{array}$ & $\begin{array}{c}2404(80.9 \%) \\
568(19.1 \%) \\
133(4.5 \%)\end{array}$ & $\begin{array}{r}23842(81.4 \%) \\
5465(18.6 \%) \\
1351(4.6 \%)\end{array}$ \\
\hline $\begin{array}{l}\text { Drugs } \\
\text { Oral steroids } \\
\text { Weak/moderate topical steroids } \\
\text { Strong/very strong topical steroids } \\
\text { Thiazides } \\
\text { Beta-blockers } \\
\text { ARBs } \\
\text { Low-dose aspirin } \\
\text { Non-aspirin NSAIDs } \\
\text { Antidepressants } \\
\text { Statins }\end{array}$ & $\begin{array}{r}939(13.3 \%) \\
812(11.5 \%) \\
1037(14.7 \%) \\
1285(18.2 \%) \\
1482(21.0 \%) \\
1188(16.9 \%) \\
1615(22.9 \%) \\
2265(32.2 \%) \\
1118(15.9 \%) \\
1664(23.6 \%)\end{array}$ & $\begin{array}{r}9784(13.9 \%) \\
8351(11.9 \%) \\
11390(16.2 \%) \\
12676(18.0 \%) \\
14837(21.1 \%) \\
11438(16.2 \%) \\
16956(24.1 \%) \\
22824(32.4 \%) \\
11996(17.0 \%) \\
16701(23.7 \%)\end{array}$ & $\begin{array}{l}257(8.7 \%) \\
301(10.1 \%) \\
420(14.1 \%) \\
679(22.9 \%) \\
806(27.1 \%) \\
144(4.9 \%) \\
\text { NA } \\
677(22.8 \%) \\
389(13.1 \%) \\
978(32.9 \%)\end{array}$ & $\begin{array}{l}2706(9.2 \%) \\
3146(10.7 \%) \\
4549(15.5 \%) \\
6093(20.8 \%) \\
8212(28.0 \%) \\
1444(4.9 \%) \\
\text { NA } \\
6670(22.8 \%) \\
4309(14.7 \%) \\
9859(33.6 \%)\end{array}$ \\
\hline $\begin{array}{l}\text { Diagnoses }^{a} \\
\text { Non-melanoma skin cancer } \\
\text { Diabetes } \\
\text { COPD } \\
\text { Alcohol-related disease } \\
\text { Moderate/severe renal disease }\end{array}$ & $\begin{array}{r}222(3.2 \%) \\
496(7.0 \%) \\
185(2.6 \%) \\
219(3.1 \%) \\
97(1.4 \%)\end{array}$ & $\begin{array}{r}644(0.9 \%) \\
4931(7.0 \%) \\
2710(3.8 \%) \\
3244(4.6 \%) \\
789(1.1 \%)\end{array}$ & $\begin{array}{l}813(27.4 \%) \\
500(16.8 \%) \\
322(10.8 \%) \\
127(4.3 \%) \\
245(8.2 \%)\end{array}$ & $\begin{array}{l}3900(13.3 \%) \\
4927(16.8 \%) \\
3753(12.8 \%) \\
1392(4.8 \%) \\
2234(7.6 \%)\end{array}$ \\
\hline $\begin{array}{l}\text { Highest education achieved } \\
\text { Short ( } \leqslant 10 \text { years) } \\
\text { Medium (11-13 years) } \\
\text { Long ( }<13 \text { years) }\end{array}$ & $\begin{array}{l}1659(23.5 \%) \\
3225(45.8 \%) \\
1931(27.4 \%)\end{array}$ & $\begin{array}{l}21450(30.4 \%) \\
30399(43.1 \%) \\
14875(21.1 \%)\end{array}$ & $\begin{array}{l}\text { NA } \\
\text { NA } \\
\text { NA }\end{array}$ & $\begin{array}{l}\text { NA } \\
\text { NA } \\
\text { NA }\end{array}$ \\
\hline
\end{tabular}


Table 2. Association between use of PDEls and risk of invasive melanoma, specified by exposure pattern and disregarding prescriptions within the year prior to the index date

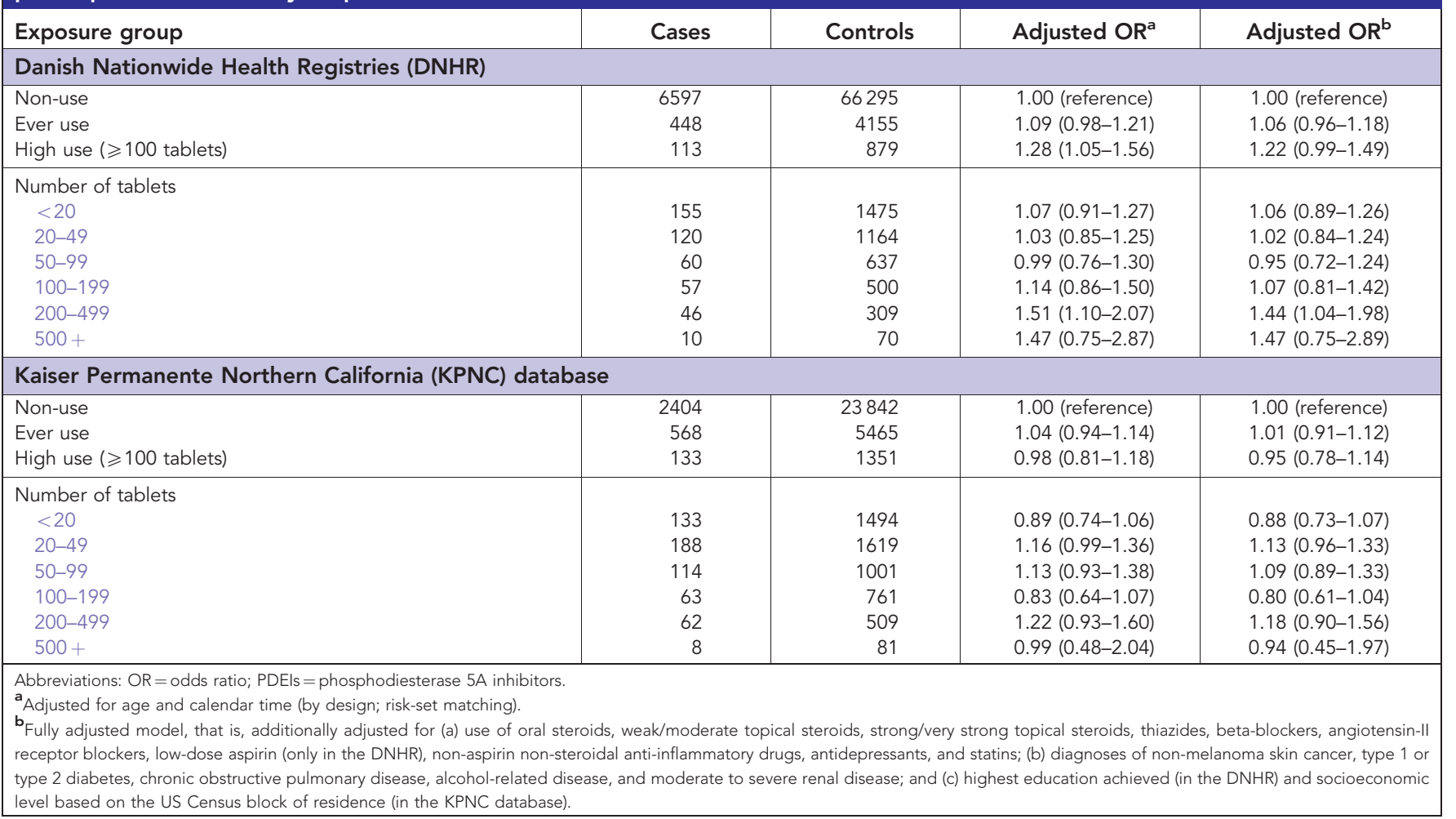

compared with no lag time (OR, 1.15). Within the KPNC database, the corresponding sensitivity analysis did not materially influence the results (ORs, 0.99 vs 0.97).

\section{DISCUSSION}

In two independently conducted large-scale case-control analyses using Danish and US data, including more than 10000 melanoma cases, we found little evidence for a causal relation between use of PDEIs and risk of melanoma. In the KPNC population, all estimates were close to unity both with increasing PDEI use and in patient subgroups. Results were generally similar in the DNHR data, although high cumulative use was associated with a moderate increase in the risk of melanoma, mainly driven by elevated risk estimates with use of $\geqslant 200$ tablets of PDEIs. Of particular note, PDEI use was associated with slightly increased risks of localised melanoma in Denmark and of melanoma in situ in the US data, whereas a decreased risk of non-localised disease was observed in both study populations. This pattern is compatible with a detection bias stemming from more intensive contact with the health-care system among PDEI users. The possibility of detection bias is further supported by the observed attenuation of associations after adjustment for education and ambulatory visits, incorporated in the analyses as markers of health-care-seeking behaviour.

In addition to its large size, the major strengths of the present study are its use of high-quality health-care data from two unique source populations, along with the use of similar study designs and data analyses in the two substudies. The two study populations differed with respect to patterns of PDEI use, melanoma incidence, and availability of data on potential confounders. Together, this heterogeneity permitted analysis of several different PDEIs, in two study settings with markedly different sun exposure, of disease ranging from in situ to advanced, and examination of a broad range of potentially confounding variables. This revealed important features such as the potential for detection bias. A mutual weakness in the two study populations was lack of data on lifestyle factors, notably sun exposure, smoking, and obesity. It is difficult to predict the direction in which these factors might bias the risk estimates; for example, smoking (Song et al, 2012) and obesity (Sergentanis et al, 2013) have been found to have opposite associations with melanoma risk. The study by Matthews et al (2016) suggested that sun exposure was associated with the use of PDEIs and confounding from sun exposure may thus have biased our risk estimates upwards. However, the only study with self-reported sun exposure information ( $\mathrm{Li}$ et al, 2014) found very similar sun exposure histories in users and non-users of PDEIs. Another caveat in our study was potential exposure misclassification due to selfmedication with PDEIs purchased over the internet (Shaeer, 2013). However, as regular users of PDEIs in both Denmark and among KPNC members have a financial incentive for obtaining PDEIs by prescription, any online purchase would result predominantly in misclassification of true users among study subjects classified as nonusers or with low-cumulative PDEI use, thereby introducing at maximum only a small bias towards the null.

Direct comparison of our findings with the study by Li et al (2014) is hampered by important differences in the analytical approach and data sources. In the Li et al (2014) study, PDEI exposure was defined as self-reported use at the start of follow up (ever or recent use compared with never use). Exposure status was not updated during follow up and no data were provided on doseresponse relationships. In contrast, our data and analytical approach were very similar to that of the Swedish study by Loeb et al, 2015. Still, an important limitation of the Swedish study was the limited exposure period based on prescription data only from July 2005 onwards. Due to the sparse exposure data, the authors used a low cut-off to define high PDEI use at only six prescriptions, corresponding to 24-72 tablets (assuming 4-12 tablets per prescription). Our results, based on the hitherto largest study of 
Table 3. Associations between high use of PDEls ( $\geqslant 100$ tablets) and risk of melanoma, by clinical stage

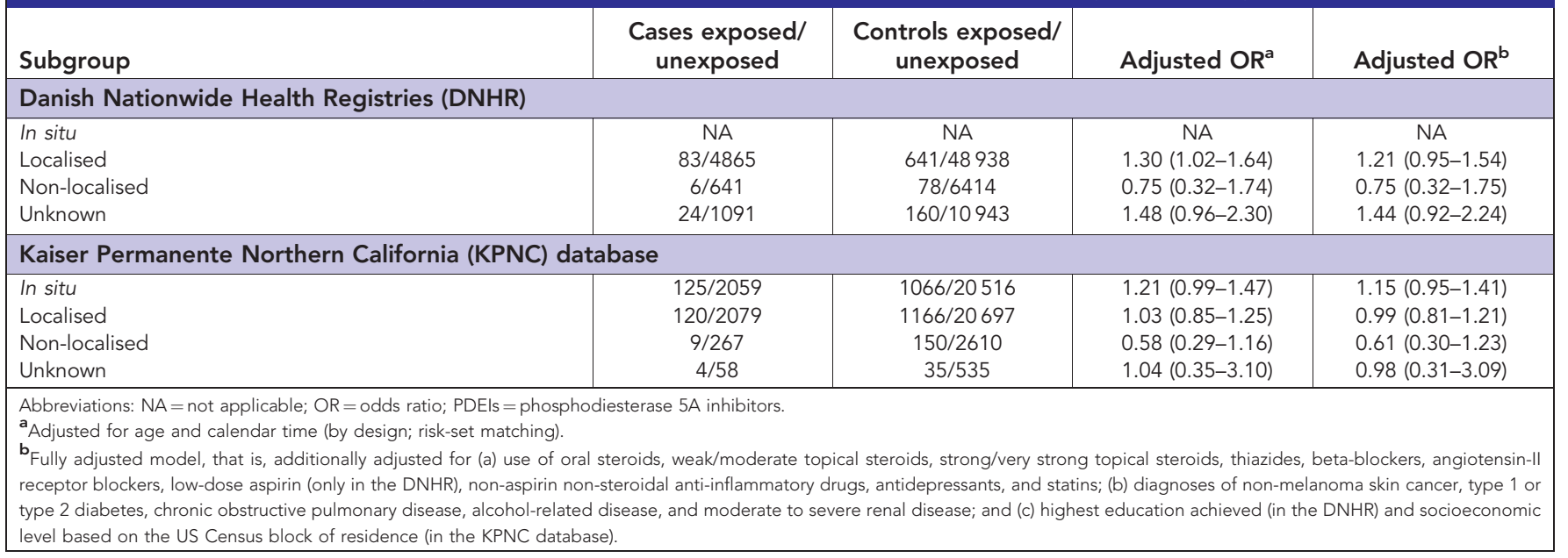

the association between PDEI use and melanoma risk, thus complement those of the (Loeb et al, 2015) and the two other previous studies (Lian et al, 2016; Matthews et al, 2016) by providing results for high cumulative use of PDEIs. Further, our study supports the notion that the slightly increased risk of notably localised melanoma observed across the studies can be attributed to residual confounding from sun exposure and health-seeking behaviour.

In conclusion, our findings provide little support for a causal association between use of PDEIs and risk of melanoma. Although we did observe marginally increased risks for melanoma in situ in the US study population and localised melanoma in the Danish population, we attribute these findings to more intensive healthcare adherence among PDEI users, and thereby to the potential for earlier melanoma detection.

\section{ACKNOWLEDGEMENTS}

Lotte Rasmussen (MScPharm, University of Southern Denmark) and Sidsel Arnspang Pedersen (MD, University of Southern Denmark) are acknowledged for valuable comments to the manuscript. Morten Olesen (University of Southern Denmark) is acknowledged for assistance with data management. The Danish study was funded by the Danish Council for Independent Research (grant 4004-00234B). The study at Kaiser Permanente Northern California was funded by the National Cancer Institute (grant R01CA098838).

\section{CONFLICT OF INTEREST}

LAH reports research support from grants from Takeda, Sanofi, and Genentech to Kaiser Permanente Research Foundation. The remaining authors declare no conflict of interest.

\section{REFERENCES}

Arozarena I, Sanchez-Laorden B, Packer L, Hidalgo-Carcedo C, Hayward R, Viros A, Sahai E, Marais R (2011) Oncogenic BRAF induces melanoma cell invasion by downregulating the cGMP-specific phosphodiesterase PDE5A. Cancer Cell 19: 45-57.

Bollag G, Tsai J, Zhang J, Zhang C, Ibrahim P, Nolop K, Hirth P (2012) Vemurafenib: the first drug approved for BRAF-mutant cancer. Nat Rev Drug Discov 11: 873-886.
Burstein HJ, Schwartz RS (2008) Molecular origins of cancer. N Engl J Med 358: 527.

Ghofrani HA, Osterloh IH, Grimminger F (2006) Sildenafil: from angina to erectile dysfunction to pulmonary hypertension and beyond. Nat Rev Drug Discov 5: 689-702.

Gjerstorff ML (2011) The Danish Cancer Registry. Scand J Public Health 39: $42-45$.

Gray-Schopfer V, Wellbrock C, Marais R (2007) Melanoma biology and new targeted therapy. Nature 445: 851-857.

Hauschild A, Grob J-J, Demidov LV, Jouary T, Gutzmer R, Millward M, Rutkowski P, Blank CU, Miller WH, Kaempgen E, Martín-Algarra S, Karaszewska B, Mauch C, Chiarion-Sileni V, Martin A-M, Swann S, Haney P, Mirakhur B, Guckert ME, Goodman V, Chapman PB (2012) Dabrafenib in BRAF-mutated metastatic melanoma: a multicentre, open-label, phase 3 randomised controlled trial. Lancet 380: 358-365.

Jensen VM, Rasmussen AW (2011) Danish Education Registers. Scand J Public Health 39: 91-94.

Kildemoes HW, Sørensen HT, Hallas J (2011) The Danish National Prescription Registry. Scand J Public Health 39: 38-41.

Lian Y, Yin H, Pollak MN, Carrier S, Platt RW, Suissa S, Azoulay L (2016) Phosphodiesterase type 5 inhibitors and the risk of melanoma skin cancer. Eur Urol; e-pub ahead of print 10 May 2016; doi: 10.1016/j.eururo.2016.04.035.

Li W-Q, Qureshi AA, Robinson KC, Han J (2014) Sildenafil use and increased risk of incident melanoma in US men: a prospective cohort study. JAMA Intern Med 174: 964-970.

Loeb S, Folkvaljon Y, Lambe M, Robinson D, Garmo H, Ingvar C, Stattin P (2015) Use of phosphodiesterase type 5 inhibitors for erectile dysfunction and risk of malignant melanoma. JAMA 313: 2449-2455.

Matthews A, Langan SM, Douglas IJ, Smeeth L, Bhaskaran K (2016) Phosphodiesterase type 5 inhibitors and risk of malignant melanoma: Matched Cohort Study using primary care data from the UK clinical practice research datalink. PLoS Med 13: e1002037.

Pedersen CB (2011) The Danish Civil Registration System. Scand J Public Health 39: 22-25.

Rothman KJ, Greenland S, Lash TL (2008) Modern Epidemiology, 3rd edn. Wolters Kluwer Health, Lippincott Williams \& Wilkins: Philadelphia, PA, USA.

Schmidt M, Pedersen L, Sørensen HT (2014) The Danish Civil Registration System as a tool in epidemiology. Eur J Epidemiol 29: 541-549.

Schmidt M, Schmidt SAJ, Sandegaard JL, Ehrenstein V, Pedersen L, Sørensen HT (2015) The Danish National Patient Registry: a review of content, data quality, and research potential. Clin Epidemiol 7: 449-490.

Selby J, Smith D, Johnson E, Raebel M, Friedman G, McFarland B (2005) Kaiser Permanente Medical Care Program. In Strom BL (ed). Pharmacoepidemiology, 4th edn. Wiley \& Sons: Chichester, West Sussex.

Sergentanis TN, Antoniadis AG, Gogas HJ, Antonopoulos CN, Adami H-O, Ekbom A, Petridou ET (2013) Obesity and risk of malignant melanoma: a meta-analysis of cohort and case-control studies. Eur J Cancer 49: 642-657. 
Shaeer O (2013) The Global Online Sexuality Survey (GOSS): the United States of America in 2011 chapter II: phosphodiesterase inhibitors utilization among English speakers. J Sex Med 10: 532-540.

Shamloul R, Ghanem H (2013) Erectile dysfunction. Lancet 381: 153-165. Song F, Qureshi AA, Gao X, Li T, Han J (2012) Smoking and risk of skin cancer: a prospective analysis and a meta-analysis. Int J Epidemiol 41: 1694-1705.
Thygesen LC, Daasnes C, Thaulow I, Brønnum-Hansen H (2011) Introduction to Danish (nationwide) registers on health and social issues: structure, access, legislation, and archiving. Scand J Public Health 39: 12-16.

(c) (1) (2) (2) This work is licensed under the Creative Commons (c) Attribution-Non-Commercial-Share Alike 4.0 International License. To view a copy of this license, visit http:// creativecommons.org/licenses/by-nc-sa/4.0/

Supplementary Information accompanies this paper on British Journal of Cancer website (http://www.nature.com/bjc) 